\title{
PERFIL DE CONSUMIDORES DE LOS NUEVOS SEGMENTOS DE MERCADO: CASO SALTILLO, COAHUILA
}

\section{CONSUMER PROFILE OF THE NEW MARKET SEGMENTS: CASE OF SALTILLO, COAHUILA}

Adriana Méndez-Wong ${ }^{1}$ * (D); Juana Alicia Villarreal-Cavazos ${ }^{2}$ (D); Elizabeth Ana Aguilar-Garcés ${ }^{3}$ (iD).

1. Universidad Autónoma de Coahuila. adrianamendezwong@uadec.edu.mx y amendezwong@hotmail.com

2. Universidad Autónoma de Coahuila. juanavillarreal@uadec.edu.mx

3. Universidad Autónoma de Coahuila. anaaguilar@uadec.edu.mx

*Autor de Correspondencia: Adriana Méndez-Wong, correo electrónico: adrianamendezwong@uadec.edu.mx

\section{RESUMEN}

A través del tiempo, los mercadólogos han utilizado técnicas de segmentación especializadas para encontrar sus mercados meta. Angus y Westbrook, (2018), (2019), (2020) y (2020) detallan dentro de las tendencias globales de consumo nuevos perfiles de clientes.

Con este trabajo se pretende abordar la propensión de prácticas de compra de los saltillense a través de un estudio exploratorio / descriptivo, con enfoque cuantitativo y utilizando estadística descriptiva y análisis factorial. Aplicando un instrumento de cuarenta y nueve ítems, con una escala de respuesta de tipo Likert del 1 al 7. La muestra es de 275 sujetos de 15 a 78 años en la ciudad de Saltillo, Coahuila y el trabajo de campo se realizó durante los meses enero y febrero del año 2020.

El análisis realizado fue el de la varianza de un factor (ANOVA) con el fin de comprobar si hay diferencias significativas entre cada una de los ítems que integran la escala. Los resultados del estudio nos permitieron comprender mejor a los consumidores que emergen en la actualidad en la ciudad de Saltillo, Coahuila.

Palabras clave: comportamiento del consumidor; segmentación; perfiles de clientes; tendencias.

Cómo citar:

Méndez-Wong, Adriana; Villarreal-Cavazos, Juana Alicia; Aguilar-Garcés, Elizabeth Ana. (2021). PERFIL DE CONSUMIDORES DE LOS NUEVOS SEGMENTOS DE MERCADO: CASO SALTILlO, COAHUILA. Revista de Investigaciones Universidad del Quindio, 33(S1), 43-55. https://doi.org/10.33975/riuq.vol33nS1.482 


\begin{abstract}
Over time, marketers have used specialized segmentation techniques to find their target markets. Angus and Westbrook, (2018), (2019), (2020) and (2020) detail new customer profiles within global consumer trends.

This work is intended to address the propensity of purchasing practices of Saltillo through an exploratory / descriptive study, with a quantitative approach and using descriptive statistics and factor analysis. Applying an instrument of forty-nine items, with a Likert-type response scale from 1 to 7 . The sample is of 275 subjects aged 15 to 78 years in the city of Saltillo, Coahuila and the field work was carried out during the months January and February of the year 2020.

The analysis carried out was that of the variance of one factor (ANOVA) in order to check if there are significant differences between each of the items that make up the scale.

The results of the study allowed us to better understand the consumers currently emerging in the city of Saltillo, Coahuila.
\end{abstract}

Keywords: consumer behavior; segmentation; customer profiles; trends.

\title{
INTRODUCCIÓN
}

En los últimos años, se encuentran investigaciones, que sugieren que el consumo tradicional está cambiando su dinámica hacia nuevos modelos de negocios alineados con el actual contexto competitivo (Santucci-Oiveira, Bauer, Tomelin , \& Lisboa -Sohn , 2019) (Menor-Campos, Hidalgo-Fernandez , \& Valverde-Roda, 2019).

La relevancia del estudio radica en aportar con evidencia empírica contribuyendo a la literatura del área del comportamiento del consumidor, específicamente en la descripción de nuevos segmentos de consumidores. Y, para alcanzar el objetivo, el trabajo se ha dividido en los siguientes apartados: primero, se presenta la revisión de la literatura describiendo los nueve constructos: Limpios, Arrendatarios, Activistas, Saludables, Digitales Expertos, Detectives, Simples, Conscientes y Desconectados; así mismo se analiza los antecedentes de investigaciones sobre el tema en cuestión; seguido de la metodología y resultados del estudio; por último, se expondrán las conclusiones.

\section{MARCO TÉORICO}

Alison Angus (2018) afirma que el 60\% de los consumidores entre 20 y 29 años están de acuerdo en que pueden hacer diferencia en el mundo a través sus acciones. Además, plantea que los defensores de la vida "limpia" son estrictos en sus opciones de abstención, ya sea en su veganismo, en evitar el alcohol o cualquier otra opción de estilo de vida, pero son influenciadores por lo que esta tendencia se extenderá a otros.

Un estudio realizado por Janssen, Busch, Rödiger y Ham (2016) entrevistando a consumidores que acudían a siete supermercados veganos en Alemania, expuso que los principales motivos para seguir una dieta vegana son en primer lugar lo relacionado con el maltrato animal (89.7\%), motivos relacionados con el bienestar personal y la salud (69.35), y motivos relacionados con el medio ambiente (46.8\%). 
El comportamiento de compra de los consumidores en relación con los productos alimentarios ha sido largamente estudiado, concluyendo que el contexto en el cual tiene lugar el nivel de información que manejan los consumidores y el uso del producto afecta a la elección del mismo. Además, autores han confirmado el impacto de la disponibilidad de alimentos saludables y bebidas no alcohólicas en las tiendas minoristas en los resultados de la salud, como la obesidad (Mhurchu, y otros, 2013)

En el mismo apartado del análisis del veganismo, Daniele Rodrigues (2019) llevó a cabo un estudio comparativo obteniendo que, los motivos de consumo que determinan la compra de carne de vacuno son diferentes; por ejemplo, en Brasil, tienen más importancia factores como "por la familia", "gusto/satisfacción”, “adecuarse a celebraciones" y "costumbre/tradición” mientras que, en España "enriquecer la dieta" y ser "saludable" son considerados más importantes.

En lo que respecta al constructo de "arrendatarios", se observa que el consumo colaborativo en los últimos años ha crecido rápidamente, como lo sostienen Ertz, Durif and Arcand (2017) al afirmar que el consumo colaborativo (CC) es un fenómeno social, en Estados Unidos, un 40\% de los adultos afirman haber participado en los esquemas de arrendamiento habilitados para la web (por ejemplo, Airbnb, Uber).

Se define el consumo colaborativo como el acceso a productos y servicios que no implican necesariamente la compra de estos o al menos la compra total; también es llamado como economía compartida por su particularidad de compartir los bienes en vez de ser único propietario. Según Cañigueral (2014) citado por el Instituto Universitaria Esumer (2018) el consumo colaborativo propone compartir los bienes frente a poseerlos, y focalizarse en poner en circulación todo eso que ya existe. Lo anterior, implica un cambio de paradigma frente al consumo ya que deja de entenderse como propiedad para convertirse como acceso y uso. En el año 2010 el consumo colaborativo se popularizó, comenzando a implementarse en diferentes países, impactando directamente al consumismo y a la adquisición de bienes materiales, gracias a sus grandes potencialidades para reducir la producción masiva e impulsar a la población a nuevas prácticas como el uso compartido de automóviles, utensilios de la vida cotidiana, vivienda etc.

El comportamiento ético de los consumidores, también conocido como el consumo político, es una elección libre por parte de los consumidores que es motivada por las preocupaciones políticas, sociales o medioambientales que se puede plasmar de dos maneras principales: al no comprar ciertos productos (boicotear) o específicamente comprar productos con un comercio justo o con etiqueta orgánica ("buycotting" en inglés) ( (Renard, 2003); (Shaw, Newholm, \& Dickinson, 2006)). Hay muchas variaciones del activismo en el consumidor (Glickman, 2009), pero en general, el propósito de los consumidores activistas es dañar o apoyar los ingresos de una organización directamente, por ejemplo, dejando de gastar o gastando dinero en un producto u organización en particular, o utilizando medios de comunicación u otros terceros para dañar o mejorar la imagen de una empresa, afectando así en última instancia a sus ingresos (Friedman, 2002 ; King, 2011). Ahora bien, los valores sociales y las preferencias políticas también influyen en las elecciones de los consumidores en forma de "boicot" - no comprar un bien o servicio - o de un "buycott" - el consumo intencional de un bien o servicio (Endres \& Panagopoulos, 2017).

Angus (2018), describe a los consumidores preocupados por su salud a causa de la creciente curiosidad con respecto a su composición genética, lo que los hace tan especiales y el gran interés en la salud y belleza personalizadas. Esto impulsado la demanda por kits de ADN para el hogar. El público objetivo 
va desde los "preocupados" y los curiosos con respecto a sus orígenes a fanáticos del estado físico y la nutrición. Empresas como 23andMe, DNAFit y AncestryDNA mapean el código genético por medio de muestras simples de sangre o saliva y explican su significado. Aunque el mercado aún se encuentra en pañales, valorado en USD 70 millones en 2015 Credence Research declara que el mercado global se encuentra en expansión y se espera que se dispare a USD 340 millones para el 2022.

Los consumidores "digitales expertos" son aquellos que acostumbran utilizar las redes sociales para solicitar recomendaciones y donde sus decisiones de compra están influenciadas por las publicaciones en redes sociales. Gómez Nieto (2018), realizó una investigación en que menciona que el influencer implica una nueva versión del líder de opinión aplicada al medio en línea, aprovechando el enorme potencial que muestran las redes sociales. En su estudio cita que el 85\% de los usuarios declaran que siguen a los influencers en las redes sociales. Además, este tipo de clientes son los considerados adoptadores tempranos, y esto incluye el uso de Realidad Aumentada, lo que les permite experimentar compras en líneas "probando antes de comprar" (Angus, 2018).

Cuando una persona se da cuenta de que su pensamiento es contradictorio con sus propias elecciones, prácticas, hábitos y rutinas cotidianas a través de una nueva perspectiva y estos viejos hábitos no se doblan a su nueva imagen interior de sí mismo, por ejemplo, como ser un consumidor ético, éste va a cambiar sus costumbres y acciones. Una persona trata de armonizar su propia imagen, y el objetivo es una autoidentidad intacta y una historia de vida equilibrada. Actuando éticamente, el consumidor sabe que actúa moralmente correctamente y de esta manera se aproxima a un mundo ético ideal (Giddens, 1991). (Spaargaren \& van Vliet, 2000) y (Niinimäki, 2010 ). Moschis \& Churchill (1979) citado por Purohit (2018), describen que el comportamiento de los consumidores está influenciado por habilidades directas e indirectas, las habilidades directas del consumidor están relacionadas con las actividades de consumo y las decisiones de compra y las habilidades indirectas incluyen la conciencia del consumidor, su conocimiento, su actitud y otros factores que influyen en la decisión de compra. Tal es el caso de los "compradores detectives" (Angus, 2018), quienes investigan todo el proceso de producción, desde el abastecimiento de materiales / ingredientes hasta la distribución, pasando por todas las partes involucradas en el proceso. Una evidencia detallada de las prácticas laborales permite a los compradores detectives entender la historia completa detrás del desarrollo y producción del producto. Este tipo de consumidores consideran las características morales de los productos en sus decisiones de compras cotidianas: compran alimentos orgánicos, usan energías renovables o boicotean prendas fabricadas bajo condiciones de trabajo dudosas. Comprar productos de comercio justo (Fair Trade en inglés) es un ejemplo destacado del comportamiento moralmente orientado del consumidor, ya que los intercambios de estos productos en el mercado implican un componente altruista (Beckert, 2006).

Para explicar las características de los "Clientes Simples y con Status" (Angus \& Westbrook, 2020) se destaca que son aquellos que rechazan los productos genéricos, favoreciendo el consumo de los productos posicionados como más simples, de vuelta a lo básico y de mejor calidad, con un nivel de estatus implícito. Como los son los productos locales, como cerveza y licores artesanales, alimentos para bebé hechos en casa y experiencias de eco-lujo. Y en relación a los "Consumidores Conscientes" son quienes buscan formas de tomar decisiones positivas sobre lo que compran y una solución al impacto negativo del consumismo global. Este enfoque respetuoso y compasivo involucra la consciencia sobre otros seres humanos, animales y el medio ambiente. El bienestar animal es el enfoque de esta tendencia. 
En Turquía se llevó a cabo un estudio sobre la creciente importancia del consumo sostenible, encontrando que la preocupación ambiental, el altruismo y la conciencia, son los factores relevante en la compra ecológica (Yarimoglu \& Binboga, 2018).

Con la presión de mantenernos conectados, se presenta una tendencia JOMO,( abreviatura de Joy of missing out), contraria a la tendencia FOMO, el temor a no participar o ser dejado de lado (FOMO "Fear of Missing Out), de clientes que responden al gozo de "desconectar" de apagar los diferentes dispositivos y desaparecer (JWT, 2012). Para desconectarse, los consumidores están escogiendo dejar de lado sus teléfonos más a menudo y enfocarse en experiencias de la vida real (2020)

\section{MÉTODO}

Es un estudio con enfoque cuantitativo, exploratorio y descriptivo. Utilizando estadística descriptiva y análisis factorial; el tamaño de la muestra es de 275 sujetos; el procedimiento de muestreo es Aleatorio simple; con un error muestral de 4.0\%; $\mathrm{p}=\mathrm{q}=0.5$; nivel de confianza 95\%; el Cuestionario está estructurado de 49 ítems con 9 dimensiones:1) consumidor limpio, 2) cliente arrendatario, 3) consumidor activista, 4) consumidor saludable, 5) cliente digital experto, 6) consumidor detective, 7) consumidor simple y con status, 8) consumidor consciente y 9) consumidor desconectado; la Escala es adaptada de la Encuesta de Tendencias de Consumo Globales 2017, 2018 y 2019 de Euromonitor International. (Angus, 2018) (Angus \& Westbrook, 2019) y (Angus \& Westbrook, 2020). Las preguntas son categóricas y la escala de respuesta es tipo Likert del 1 al 7: Donde 1 es totalmente en Desacuerdo, 4 Ni de acuerdo, ni en desacuerdo y 7 Totalmente de Acuerdo. El trabajo de campo se llevó a cabo durante enero y febrero del año 2020, en la Ciudad de Saltillo, Coahuila México.

Considerando la información que se recolectó en este trabajo de investigación y las variables de segmentación que fueron establecidas en el instrumento de recolección de datos, se consideró pertinente analizar la información obtenida mediante una prueba de análisis de la varianza de un factor (ANOVA), mediante la que se analizaron, de manera sistemática, las medias de la muestra para determinar si los datos provienen de la misma población objeto de estudio, y determinar, de esta manera, el nivel de significancia existente entre las variables analizadas y sus respectivos factores de segmentación. Enfatizando que se cumple con las condiciones para usar ANOVA de un factor, pues la variable dependiente es cuantitativa (escalar) y la variable independiente es nominal (Género).

\section{HALLAZGOS}

Los datos utilizados en este trabajo provienen de una encuesta, cuya ficha técnica se encuentra en la Tabla 1. El instrumento estaba estructurado en dos grandes bloques. El primero de ellos detallaba características de los entrevistados (género, estado civil, nivel de estudios, ocupación) y el segundo bloque se centraba en conocer las percepciones y prácticas de compra de los saltillenses.

Tabla 1. Ficha técnica del estudio

\begin{tabular}{|l|l|}
\hline Población & Personas de 15 a 78 años, de ambos sexos en Saltillo, Coahuila, México \\
\hline Tamaño muestra & 275 encuestas personales \\
\hline Error muestral & $4.0 \% ; \mathrm{p}=\mathrm{q}=0.5 ;$ nivel de confianza 95\% \\
\hline Procedimiento de Muestreo & Aleatorio simple \\
\hline Método de trabajo de campo & Encuesta Auto Administrada \\
\hline \multicolumn{2}{|c|}{ Fuente: Elaboración propia } \\
\hline
\end{tabular}


Los valores especificados en la Tabla 2 muestran un Alfa de Cronbach's de 0.771 que de acuerdo con Hair señalan una alta confiabilidad de los datos (Hair, Anderson, Tatham, \& C., 2001; Nunnaly, 1987).

Tabla 2. Estadísticos de fiabilidad Alfa de Cronbach

\begin{tabular}{cc}
\hline Alfa de Cronbach & N de elementos \\
\hline .771 & 49 \\
\hline Fuente: Elaboración propia
\end{tabular}

\section{Análisis de la Muestra}

El perfil demográfico de los encuestados es que sus edades oscilan entre 15 y 78 años; el $46.8 \%$ de los entrevistados son hombres y el 53.2\% son mujeres; el $2.5 \%$ posee estudios de posgrado, un $43 \%$ cuenta con estudios de Licenciatura, el 33\% bachillerato, 13\% secundaria y $8.5 \%$ nivel primaria; en relación con el estado civil el $68 \%$ manifiesta ser soltero, un $24 \%$ casado, un $4 \%$ divorciado y un $4 \%$ otros.

\section{Análisis Descriptivo y factorial}

El análisis que se utilizó en este estudio y que se aplicó a la información obtenida fue el de la varianza de un factor (ANOVA), para poder comprobar mediante él si existen diferencias significativas entre cada una de las cuarenta y nueve ítems o preguntas que integran la escala. Por lo tanto, los resultados obtenidos de los nueve perfiles de los consumidores procedentes de Saltillo Coahuila se presentan en las tablas que se muestran a continuación:

Tabla 2. Situación media de los consumidores respecto a la Escala ${ }^{1}$.

\begin{tabular}{|c|c|c|}
\hline Variables & Medias & Significancia \\
\hline \multicolumn{3}{|l|}{ Limpios } \\
\hline Prefiero gastar mi dinero en experiencias en lugar de en cosas & 4.69 & $.008 * * *$ \\
\hline Prefiero alimentos que no sean de origen animal & 3.49 & $.000 * * *$ \\
\hline Me gusta pasar tiempo con la familia & 5.86 & $.000 * * *$ \\
\hline Prefiero las bebidas sin alcohol & 4.19 & .208 \\
\hline $\begin{array}{l}\text { Me gusta quedarme en casa y relajarme en lugar de ir a un bar o club } \\
\text { nocturno. }\end{array}$ & 5.34 & .794 \\
\hline \multicolumn{3}{|l|}{ Arrendatarios } \\
\hline Prefiero rentar vestido de noche y traje en lugar de comprar. & 3.85 & $.000 * * *$ \\
\hline Acostumbro utilizar aplicaciones de taxi como Uber, Cabify, Didi & 4.13 & .942 \\
\hline Me interesa hospedarme mediante la plataforma Airbnb & 3.57 & $.033 * *$ \\
\hline Estaría dispuesto a rentar aparatos para hacer ejercicios & 3.64 & .894 \\
\hline $\begin{array}{l}\text { Siento que puedo hacer una diferencia en el mundo a través de mis } \\
\text { acciones. }\end{array}$ & 5.67 & $.002 * * *$ \\
\hline Prefiero gastar mi dinero en experiencias en lugar de en cosas & 4.68 & $.008 * * *$ \\
\hline $\begin{array}{l}\text { Compro un artículo o servicio al menos } 1 \text { a } 2 \text { veces al mes o más con mi } \\
\text { teléfono celular }\end{array}$ & 3.30 & .113 \\
\hline Prefiero ver o probar antes de comprar & 5.40 & .932 \\
\hline
\end{tabular}


Prefiero comprar en línea ya que puedo comprar en cualquier momento desde cualquier lugar

4.19

.466

Activistas

He utilizado plataformas para peticiones o apoyo a ciertas causas como Change. org y Avaaz.org

En línea, he dado mi opinión acerca de alguna marca.

4.11

.276

Visito o actualizo mis redes sociales al menos una vez a la semana

6.10

.122

Cuando veo una queja en redes sociales respecto a una marca, Investigo a

la marca antes de comprarla

4.99

$.046 * *$

Cuando veo una queja en redes sociales respecto a una marca, refuerzo el mensaje dándole like o compartiéndolo.

Compraría o boicotearía marcas con base a sus valores corporativos.

\section{Saludables}

3.63

$.013 * *$

Si pudiera me realizaría un examen genético para conocer el riesgo de desarrollar enfermedades hereditarias o hacer mejoras en el estilo de vida en base a sus hallazgos.

\section{Digitales expertos}

Compro un artículo o servicio al menos 1 a 2 veces al mes o más con mi teléfono celular

Prefiero ver o probar antes de comprar

Prefiero comprar en línea ya que puedo comprar en cualquier momento desde cualquier lugar

Me gusta la experiencia de comprar en tienda física

\section{Detectives}

Prefiero comprar en línea en tanto cuente con información, comparación y opiniones a la mano

Solo compro de empresas y marcas en las que confío

Me gusta conocer la historia completa detrás del desarrollo y producción del producto que voy a comprar

Procuro comprar productos de empresas que son honestas y reales.

\section{Simples y con Status}

Prefiero comprar productos simples, básicos y de buena calidad

Me gusta comprar productos locales en lugar de importados

En mi familia se prefiere los alimentos hechos en casa en lugar de comprarlos

Acostumbro elaborar mis propios productos de belleza y de higiene personal de origen natural

Prefiero los remedios herbales tradicionales y las alternativas con ingredientes naturalmente saludables.

Leo las etiquetas de las comidas empaquetadas para identificar nutrientes (artificiales y/o naturales, colesterol, sodio o sal, conservadores) 
Busco ingredientes totalmente naturales cuando compro productos para el cuidado de la piel

\subsection{3}

$.051 *$

\section{Conscientes}

Considero que comer carne es necesario para una buena nutrición.

$5.19 \quad .002 * * *$

No consumo carne solo cuando no tengo dinero para comprarla

4.10

.652

Prefiero una dieta con base en vegetales

3.20

.645

No deben realizar pruebas con animales en productos cosméticos y farmacéuticos

En productos para el cuidado del cabello y piel procuro que contenga colágeno o lanolina

\subsection{6}

$.005 * * *$

Busco productos que muestren la etiqueta "criado al aire libre" en productos frescos

La etiqueta "no probado en animales, fabricado sin crueldad y/o 100\% vegano" influye en mis opciones de compra en productos para el cuidado del cabello, piel, cosméticos

\section{Desconectados}

Procuro reducir mi tiempo en línea en favor de experiencias de la vida real

Últimamente no comparto fotos o estados en las redes sociales

A veces siento estrés digital, por el tiempo que paso en redes sociales

Estoy intentando desconectarme, escogiendo dejar de lado mi teléfono celular

Internet aumenta mi nivel de estrés diario

En general, me encuentro buscando maneras de simplificar mi vida

${ }^{1}$ En una escala de $1=$ total desacuerdo a $7=$ total acuerdo

Nota Diferencias estadísticamente significativas: $(*): \mathrm{p}<0.1 ;(* *): \mathrm{p}<0.05 ;(* * *): \mathrm{p}<0.01$.

\section{Fuente: Elaboración propia}

En la tabla 2 se observa que las cuatro principales variables en orden de importancia son: Prefiero comprar en línea ya que puedo comprar en cualquier momento desde cualquier lugar con una media de 6.11 en una escala de $1=$ total desacuerdo a 7=total acuerdo como límites, seguida de la variable Visito o actualizo mis redes sociales al menos una vez a la semana con una media de 6.10 , Prefiero comprar productos simples, básicos y de buena calidad, con una media de 5.91 y por último la variable Me gusta pasar tiempo con la familia con una media de 5.86 .

Además se aprecia que son diecinueve las variables en las que existen diferencias estadísticamente significativas: Prefiero gastar mi dinero en experiencias en lugar de en cosas, Prefiero alimentos que no sean de origen animal, Me gusta pasar tiempo con la familia, Prefiero rentar vestido de noche y traje en lugar de comprar, Me interesa hospedarme mediante la plataforma Airbnb, Siento que puedo hacer una diferencia en el mundo a través de mis acciones, Prefiero gastar mi dinero en experiencias en lugar de en cosas, Cuando veo una queja en redes sociales respecto a una marca, Investigo a la marca antes de comprarla, Compraría o boicotearía marcas con base a sus valores corporativos, Si pudiera 
me realizaría un examen genético para conocer el riesgo de desarrollar enfermedades hereditarias o hacer mejoras en el estilo de vida en base a sus hallazgos, Procuro comprar productos de empresas que son honestas y reales, Me gusta comprar productos locales en lugar de importados, Acostumbro elaborar mis propios productos de belleza y de higiene personal de origen natural, Prefiero los remedios herbales tradicionales y las alternativas con ingredientes naturalmente saludables, Busco ingredientes totalmente naturales cuando compro productos para el cuidado de la piel, Considero que comer carne es necesario para una buena nutrición, No deben realizar pruebas con animales en productos cosméticos y farmacéuticos, En productos para el cuidado del cabello y piel procuro que contenga colágeno o lanolina y La etiqueta "no probado en animales, fabricado sin crueldad y/o 100\% vegano" influye en mis opciones de compra en productos para el cuidado del cabello, piel, cosméticos.

Se lleva a cabo un análisis comparativo en porcentajes con el programa IBM SPSS, y a continuación se presentan los resultados:

En relación a las características de los clientes "Limpios" se encontró que un 56\% de la muestra está de acuerdo con preferir gastar en experiencias en lugar de objetos tangibles; un 27\% elige alimentos que no sean de origen animal, en comparación de un 51\% que no está de acuerdo con dicha afirmación; un $80 \%$ es hogareño, pues disfruta pasar tiempo con la familia y un $65 \%$ prefiere quedarse en casa en lugar de ir a un bar; al cuestionarles si prefieren las bebidas sin alcohol, el 44\% dijo que si, y un 37\% manifestó lo contrario.

Por lo que se refiere a los clientes "Arrendatarios" se encontró que un 30\% de la muestra está de acuerdo en preferir rentar un vestido o traje de noche en lugar de comprar; un 30\% acostumbra utilizar aplicaciones de taxi como Uber, Cabify, Didi, en comparación de un $40 \%$ que no está de acuerdo con dicha afirmación y un $30 \%$ es indeciso en esta afirmación; al cuestionar si estarían dispuestos a hospedarse mediante la plataforma Airbnb un 34\% está de acuerdo, mientras que un 46\% todavía prefiere los hoteles tradicionales; un 39\% responde que si rentaría aparatos de ejercicios en lugar de comprarlos; un $77 \%$ de la muestra considera que sus acciones pueden hacer la diferencia y un $56 \%$ menciona que prefiere gastar en experiencias que en productos.

Acerca de los clientes "Activistas" se encontró que un 24.7\% de la muestra está de acuerdo en haber utilizado plataformas en internet para pedir o apoyar ciertas causas; al cuestionarles si acostumbran en línea dar su opinión sobre las marcas, los resultados se dividen pues un 48\% está de acuerdo, en comparación de un $42.2 \%$ que no está de acuerdo en emitir sus opiniones y un $9.8 \%$ es indeciso; al preguntarles si revisan o actualizan sus redes sociales cuando menos una vez por semana , un $67.2 \%$ está de acuerdo, mientras que un $22.9 \%$ no está dedicado a monitorear sus medios sociales; un $61.1 \%$ responde que investiga las marcas antes de comprarla cuando ve quejas de ellas en las redes sociales; un $37 \%$ de la muestra refuerza el mensaje dándole like o compartiéndolo, cuando ve una queja en redes sociales con respecto a una marca; y por último un $34.5 \%$ está dispuesto a comprar o boicotear marcas con base a sus valores corporativos.

Por lo que se refiere a los clientes preocupados por el bienestar físico, al cuestionarles si pudieran se realizarían un examen genético para conocer el riesgo de desarrollar enfermedades hereditarias, un $65.8 \%$ manifestó estar de acuerdo, un 11.6 es indeciso en su respuesta y un $22.6 \%$ prefiere no conocer el riesgo.

Por otra parte, en el constructo de los clientes "Digitales expertos" un 57.8\% no está de acuerdo con la 
aseveración de comprar un artículo al menos 1 a 2 veces al mes con teléfono celular, mientras que un $13.1 \%$ es indeciso, y un $29.1 \%$ acostumbra a realizar compras en línea vía teléfono celular. Además un $69.5 \%$ manifiesta preferir ver o probar antes de comprar; un $40.4 \%$ de los participantes afirma preferir comprar en línea por la facilidad de realizarlo desde cualquier lugar y un $79.6 \%$ disfruta de comprar en tiendas físicas.

Referente a los compradores "detectives" un 70.6\% afirma que está de acuerdo en solo comprar a empresas y marcas confiables; un 57.4\% le interesa conocer la historia detrás de las marcas que consume y un $80 \%$ de los entrevistados aseguran procurar comprar productos de empresas que se perciben como honestas y reales.

En el renglón de los consumidores "simples" un 84\% prefiere comprar productos simples, básicos y de buena calidad; además el $51 \%$ tiene características de ser personas etnocéntricas; un 40\% ha comprado cerveza o licor artesanal y un 70\% consume café nacional en lugar de extranjero; se confirma que son hogareños pues el $84 \%$ antepone la comida casera; un 24\% tiene rasgos de ser eco-mixer pues elabora sus propios productos de belleza y de higiene personal con ingredientes de origen natural; un $38 \%$ utiliza los remedios herbales y un $48 \%$ compra cosmética natural. Cabe señalar que un 54\% acostumbra leer las etiquetas de las comidas empaquetadas para identificar nutrientes.

En cuanto al perfil de cliente "conscientes" un 35\% de los entrevistados elige dieta con base en vegetales; un 32\% busca productos que muestren la etiqueta "criado al aire libre"; el 63\% de los entrevistados se oponen a las pruebas con animales en productos cosméticos- farmacéuticos y busca etiquetas de $100 \%$ vegano en los productos para el cuidado del cabello, piel y cosmético. Esta cifra es muy superior al 29\% obtenido en la encuesta de belleza 2018 realizada por la empresa Eurmomonitor International (Angus, 2018).

Por último en el análisis de rasgos de clientes "desconectados", un 58.5\% procura disfrutar mas de experiencias reales que de virtuales; en tanto el $46.6 \%$ ha disminuido el compartir fotos o estados en las redes sociales; por otro lado, el $23.6 \%$ asevera sentir estrés digital diario por el tiempo que pasa en redes sociales mientras que un 48.2\% no siente presión ni estrés; un 44.3\% considera que en lo general se encuentra buscando la forma de hacer más simple su vida.

\section{DISCUSIÓN Y CONCLUSIONES}

Con base en los estudios previos y los resultados del estudio nos permitieron comprender mejor a los consumidores que emergen en la actualidad en la ciudad de Saltillo, Coahuila.

Cabe destacar que en el mercado saltillense prevalece un estilo de vida tradicional y conservador, pasando tiempo en familia y sin lujos. Pero los hallazgos nos permiten inferir que se presentan rasgos de consumidor consciente o responsable, y lo demuestran con una predisposición de consumo de compra de productos simples, de vuelta a lo básico y de mejor calidad, de procedencia local, alimentos hechos en casa y productos de belleza artesanales. Además más del 50\% está preocupado por la transparencia de los ingredientes y acostumbra leer las etiquetas de los productos que compra. Les gusta realizar compras en línea y en cuanto al uso de plataformas digitales para "rentar en lugar de comprar" como Airbn y Uber, los saltillenses están tomando decisiones con cautela, pero perciben los beneficios económicos que genera la renta de productos dentro de la economía compartida. 
Se encuentra, además, que, en el segmento de consumidor activista, su participación se vuelve más dinámica a través de medios digitales ya sea apoyando causas sociales o considerando la opinión sobre alguna marca en específico, aun cuando su dedicación en estos medios sea con una visita o actualización de sus redes, se limita a mínimo, una vez a la semana, por tanto, existe una congruencia entre la "desconexión" de los consumidores y la participación de éstos en los medios digitales al hacer uso limitado de dispositivos electrónicos.

La tendencia de pertenecer a un estilo de vida sano, ha llevado a los consumidores del segmento saludable a indagar sobre métodos que puedan analizar a detalle sus estructuras físicas para poder adecuar su imagen a estándares de belleza lo más natural posible, sin dejar de lado, situaciones futuras de salud, por lo que conocer los factores genéticos que puedan arrojar información sobre enfermedades hereditarias es prioridad para este nicho.

El cliente digital, se convierte en un consumidor experto en ambientes digitales, por lo que un teléfono inteligente le da acceso a un mercado global desde la comodidad de su hogar o desde el sitio donde se encuentre, y con base a los resultados expuestos, se concluye respecto a este segmento que, si bien, no existen fronteras para adquirir diversos productos y servicios alrededor del mundo, la fascinación por testearlos es una experiencia que prefieren seguir experimentando.

La tendencia de saber que comemos, que vestimos o usamos, lleva a los consumidores detectives a realizar un exhaustivo análisis de los productos que usa, por lo que una vez más se encuentra coincidencia con los resultados del segmento consciente, al investigar desde los componentes de un producto, hasta las condiciones laborares bajo las cuales se transforma. Así mismo, concuerda con el segmento de consumidores simples, que prefieren productos más simples como los artesanales o hechos en casa, en los que además de cuidar el estilo de vida saludable, se fomenta la participación de los miembros de la familia en la elaboración de sus propios productos.

En cuanto a la dimensión consciente, los consumidores se interesan y educan cada vez más sobre el origen de los productos que consumen, con tendencia a hábitos alimenticios más cuidados y más naturales. Buscando que las etiquetas o las propias empresas logren mostrarse transparentes respecto a sus procesos de transformación y con el mínimo impacto en el medio ambiente, al igual que el maltrato animal se vea reducido a cero, considerando que, de no ser empáticos entre ellos, tenderán a emigrar al segmento de consumidores activistas.

Relacionado al segmento desconectado, los consumidores están tratando, en la medida que les es posible de controlar el uso de sus dispositivos, tratando de crear experiencias que les permitan vivir fuera de redes sociales, en una realidad más familiar y natural, sin compartir esos momentos en sus redes sociales, creando memorias invaluables para ellos mismos.

Por lo que los resultados confirman empíricamente la tendencia de conductas en los clientes, que trae consigo nuevos segmentos de consumo, por lo que se coincide con los estudios por Janssen, Busch, Rödiger y Hamm (2016) a través del análisis de los ítems respecto a la tendencia de los consumidores preocupados por situaciones como el maltrato animal y una vida saludable. Concordando también en lo propuesto por Moschis \& Churchill (1979) y Purohit (2018), que la conciencia del consumidor, conocimiento y actitud, influyen en las decisiones de compra.

La presente investigación proporciona resultados que pueden ser un punto de partida para investigaciones 
futuras, cuyo interés sea profundizar en estas experiencias y ampliar enriqueciendo el tema de las tendencias de consumo y la generación de nuevos perfiles de clientes.

Se reconoce que el presente trabajo es de carácter exploratorio y tiene limitaciones dado que la muestra es muy amplia en el rango de la edad, requiriendo ampliar el tamaño de la muestra para que los resultados sean representativos. El trabajo de campo se llevó a cabo de enero a febrero del 2020, y se considera que a causa de la contingencia, las percepciones y estilo de vida de los saltillenses han cambiado, así como sus prioridades.

\section{REFERENCIAS}

1. Angus, A. (2018). Las 10 principales tendencias globales de consumo para 2018. Londres: Euromonitor International.

2. Angus, A. (2018). Las 10 principales tendencias globales para 2018. USA: Euromonitor International.

3. Angus, A. (2020). How is COVID-19 affecting the top 10 Global Consumer Trends 2020? Estados Unidos: Euromonitor International. Recuperado el Septiembre de 2020

4. Angus, A., \& Westbrook, G. (2019). Las 10 principales tendencias globales de consumo para 2019. Euromonitor International.

5. Angus, A., \& Westbrook, G. (2020). Las diez principales tendencias globales de consumo para 2020. Euromonitor International.

6. Beckert, J. (2006). The Moral Embeddedness of Markets. In Clary, J., Dolfsma, W. and Figart, D. M. (Eds.), Ethics and the Market: Insights from Social Economics. Londres: Routledge.

7. Cañigueral, A. (16 de Agosto de 2014). El futuro nunca estuvo tan presente. Consumo colaborativo. Obtenido de https://es.slideshare.net/acanyi/elfuturo-nunca-estuvo-tan-presente-consumocolaborativo-leaners-magazine

8. Endres, K., \& Panagopoulos, C. (2017). Boycotts, Buycotts, and Political Consumerism in America. Research and Politics.

9. Ertz, M., Durif, F., \& Arcand, M. (2017). An analysis of the origins of collaborative consumption and its implications for marketing. Academy of Marketing Studies Journal, 21(1), 1-17. Obtenido de https://papers.ssrn.com/sol3/papers.cfm?abstract_id=2799862

10. Giddens, A. (1991). Modernity and Self-Identity. Cambridge: Polity.

11. Glickman, L. B. (2009). Buying power: A history of consumer activism in America. Chicago: University of Chicago Press.

12. Gómez Nieto, B. (2018). El Influencer: herramienta clave en el contexto digital de la publicidad engañosa. Methaodos. Revista de Ciencias Sociales, 149-156.

13. Hair, J. F., Anderson, R., Tatham, R. L., \& C., B. W. (2001). Analisis Multivariado (5a. edición). Barcelona España: Ed. Prentice Hall.

14. Institución Universitaria Esumer. (2018). Consumo Colaborativo. Revista Mercatec, 8590. Obtenido de http://repositorio.esumer.edu.co/bitstream/esumer/1913/1/Consumo\%20 colaborativo.pdf

15. Janssen, M., Busch, C., Rödiger, M., \& Hamm, U. (Octuber de 2016). Motives of consumers following a vegan diet and their attitudes towards animal agriculture. Appetite, 105, 643-651. doi:https://doi.org/10.1016/j.appet.2016.06.039

16. JWT. (2012). Fear Of Missing Out (FOMO). JWT, p. 14.

17. Kotler, P., \& Keller, k. (2012). Dirección de Marketing. México: Pearson Educación.

18. Landero, Hernández, R., \& González Ramírez, M. (2007). Estadística con SPSS y Metodología de la Investigación. México: Trillas.

19. Mc Daniel, C. J., \& Gates, R. (2016). Investigación de Mercados (Vol. 10a edición ). México: Cengage Learning Editores, S.A. de C.V. .

20. Menor-Campos, A., Hidalgo-Fernandez , A., \& Valverde-Roda, J. M. (2019). La economía colaborativa: análisis conceptual. Espacios, 30-36.

21. Mhurchu, N., Vandevijvere, S., Waterlander, W., Thornton, L. E., Kelly, B., Cameron, A. J., . . . Swinburn, B. (2013). Monitoring the availability of healthy and unhealthy foods and non-alcoholic 
beverages in community and consumer retail food environments globally. Obesity Rewiews, 108119. doi: https://doi.org/10.1111/obr.12080

22. Moschis, G. P., \& Churchill, G. A. (1979). An analysis of the adolescent consumer . The Journal of Marketing, 40-48.

23. Niinimäki, K. (2010 ). Eco-Clothing, Consumer Identity and Ideology. Sustainable Development , $150-162$.

24. Nunnaly, J. C. (1987). Teoría Psicométrica. México: Ed. Trillas.

25. Purohit, H. (2018). Consumer Attitude and Buying Intention of Eco-Friendly Products . Asian Resonance, 187-191.

26. Renard, M. (2003). Fair trade: quality, market and conventions . Journal of Rural Studies, 87-96.

27. Rodrigues, D. (2019). Actitudes de los consumidores en las decisiones de compra y consumo de carne de vacuno. Obtenido de Dialnet: https://dialnet.unirioja.es/servlet/tesis?codigo $=265858$

28. Santucci-Oiveira, B., Bauer, J. E., Tomelin, C. A., \& Lisboa -Sohn, A. P. (2019). Economía compartida: un estudio sobre Airbnb. Estudios y perspectivas en turismo, 28, 636-651.

29. Shaw, D., Newholm, T., \& Dickinson, R. (2006). Consumption as voting: an exploration of consumer empowerment . European Journal of Marketing , 1049-1067.

30. Spaargaren, G., \& van Vliet, B. (2000). Lifestyles, consumption and the environment: the ecological modernisation of domestic consumption. In Ecological Modernisation around the World: Perspectives and Critical Debates. Londres: Essex.

31. Yarimoglu, E., \& Binboga, G. (2018). Understanding sustainable consumption in an emerging country: The antecedents and consequences of the ecologically conscious consumer behavior model. Business Strategy and the Environment, 642-651. doi: https://doi.org/10.1002/bse.2270 